\title{
The effect of fluid flow and heat transfer in a planar $T$ - channel with rotating cylinder
}

\author{
Palraj Jothiappan ${ }^{1}$ \\ \{palrajpsg@gmail.com\} \\ ${ }^{1}$ Department of Mathematics, PSG College of Technology, Peelamedu, Coimbatore, 641 004, TamilNadu, \\ India.
}

\begin{abstract}
The present study numerically investigates, two-dimensional laminar fluid flow and heat transfer in a planar right angled T-channel with an adiabatic rotating circular obstacle situate in the junction. The effect of fluid flow and heat transfer characteristics have been numerically explored for the range of Reynolds number $(10 \leq R e \leq 200)$ and Prandtl number of 0.71 with different cylinder rotation angle $(-5 \leq \omega \leq 5)$. The streamline and isotherm profiles have been used to explain the flow and thermal fields, respectively.
\end{abstract}

Keywords: T-channel, Rotating obstacle, Finite element method, Navier-Stokes equations and Recirculation length

\section{Introduction}

The Navier-Stokes equations and discretization methods are frequently used in computational fluid dynamics models. Methods such as finite element and finite volume analysis are two examples [2]. This equation is one of the most important ones in applied mathematics. Among the many uses for T-channel flow are irrigation, wastewater treatment, flood water conveyance and control, piping and ventilation systems, biomedical applications, pipelines for liquids and gases, polymer and pharmaceutical companies, and so on and so forth. The branching $\mathrm{T}$-junction, the impacting $\mathrm{T}$-junction, and the mixing $\mathrm{T}$-junction are three of the most commonly used flow designs. We're currently looking at the flow through a branching T-junction. A wide range of biomedical and technological applications require right angle Tchannels for heat and fluid flow. Nontrivial patterns of flow make the dynamics of such flow difficult and uncertain. It is extremely difficult to predict the flow pattern through branches under certain pressure drops because the flow resistances of different branches determine the flow distribution.

According to Hayes et al. [5], the flow in a planar branch with constant outlet pressures was studied. In a horizontal T-channel, Khandelwal et al.[7] investigated the power-law nonNewtonian fluid flow properties in the laminar regime. They discovered that for a given power-law index, the length of the secondary branch's recirculation zone increased as the Reynolds number increased. The numerical simulation of a viscous, incompressible fluid starting up in a T-channel was studied by Moshkin et al.[10]. T-junction flow is re-established after a steady pressure drop is imposed between the entry and exit points. Before a steady 
state distribution can be achieved, a start-up flow can pass through several regimes.

Bifurcation and mixing are critical in a wide range of applications, including piping systems, compact heat transfer, cardiovascular fluid mechanics, and electronic component cooling. It has been demonstrated by a number of experimental groups [6,9]. Convective heat transfer in the 90-degree bifurcation was investigated by Khodadadi et al.[8] for a wide range of flow rates and Reynolds numbers using air as the working fluid. Laminar forced convection in planar (two-dimensional) T-channels was studied by El-Shaboury et al.[3]. They found that branch size had little effect on the wall flow parameters on the run side. In an experimental study, Kawashima et al.[6] looked at the turbulent heat transfer in a right-angled juncture. The axial fluctuation of the local Nusselt number was significantly influenced by the crosssectional area and flow rate ratios, whereas the Reynolds number had a minor impact.

An adiabatic rotating circular cylinder situated at the junction of a T-channel is the focus of this study, which aims to investigate laminar flow and heat convection over a Reynolds number range of 10 to 200. Air has a Prandtl value of 0.71 when used as the working fluid in this experiment. Based on the authors' own research and the previously mentioned review of the literature. The lack of such results prompted the current investigation.

\section{Problem description}

\subsection{Geometry of the computational domain}

An illustration of the physical problem depicted in Figure 1 is shown here. The flow characteristics determine the minimum length of the bifurcation channel required to ensure a fully developed flow at the outlets. According to Shah and London [14], the length of a Newtonian-flowing pipe is determined by

$$
\frac{L}{H}=\frac{0.315}{0.068 R e+1}+0.044 R e
$$

The Reynolds number is defined as: $R e=\frac{u_{0} H}{v}$. The width of primary and secondary branches is $\mathrm{H}$. The downstream length between the junction and the primary barnch $L_{2}$ is $30 \mathrm{H}$, whereas $10 \mathrm{H}$ is the distance between inflow and channel's junction $L_{1}$. The length of secondary branch $L_{3}$ is $25 \mathrm{H}$. The cylindrical obstacle diameter is $\mathrm{D}=0.4$. The cylinder's diameter is picked to retain the $\mathrm{H} / \mathrm{D}$ ratio in the proper range, ensuring that the flow in the downstream channel remains stable Singha et al. [12]. The distance from the cylinder center to the inlet plane is $10.5 \mathrm{H}$. The upper branch right side surface $\Gamma_{4}$ and side branch lower surface $\Gamma_{7}$ are kept at a temperature that is greater than the inflow temperature, the other wall boundarys $\Gamma_{2}, \Gamma_{5}$ and $\Gamma_{8}$ are assumed as adiabatic with no slip boundary. The inlet $\Gamma_{1}$, a uniform temperature and a fully developed parabolic velocity are imposed and at the outlet boundarys $\Gamma_{3}$ and $\Gamma_{6}$ pressure is zero. Review of related research in the confined domain $[5,7,10]$, thorough flow and heat transfer analyses were carried out here at a blockage ratio of $20 \%$. 

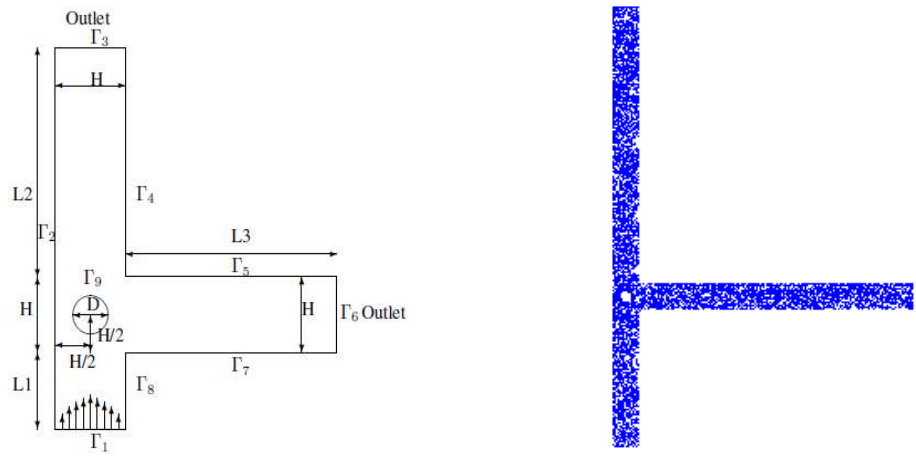

Fig 1: Flow in a T-channel, schematic diagram, Fig 2: Mesh Distribution

\subsection{Governing equations}

For two-dimensional, incompressible, isothermal, laminar, and unsteady cases, the compact version of the governing equations is as follows:

Equation of Continuity

Equation of momentum

$$
\nabla \cdot \mathbf{u}=0
$$

$$
\frac{\partial \mathbf{u}}{\partial t}+\mathbf{u} \cdot \nabla \mathbf{u}=-\nabla p+\frac{1}{R e} \nabla^{2} \mathbf{u}
$$

Equation of Energy

$$
\frac{\partial c}{\partial t}+\mathbf{u} \cdot \nabla c=\frac{1}{R e P r} \nabla^{2} c
$$

where $\mathrm{c}$ is temperature.

\subsection{Boundary conditions}

Our problem's boundary conditions are listed below,

The temperature is homogeneous and the velocity is unidirectional parabolic at the channel inlet $\Gamma_{1}$

$$
u=u_{0}, \quad v=0, \quad c=0
$$

On the left bounday $\Gamma_{4}$ of main branch and bottom $\Gamma_{7}$ of side branch are assumed temperature is constant

$$
u=0, \quad v=0, \quad c=1
$$

On the walls $\Gamma_{2}, \Gamma_{5}$ and $\Gamma_{8}$ (except the above), the assumption is that the wall is adiabatic with no-slip conditions,

$$
u=0, \quad v=0, \quad \frac{\partial c}{\partial n}=0
$$

where $n$ denote the surface normal directions

The stated velocity component with adiabatic wall boundary condition is employed on the 
cylinder surface $\Gamma_{9}$,

$$
u=-\omega\left(y-y_{0}\right), \quad v=\omega\left(x-x_{0}\right), \quad \frac{\partial c}{\partial n}=0
$$

At the out flow boundary main branch $\Gamma_{3}$,

$$
\frac{\partial u}{\partial y}=0, \quad \frac{\partial v}{\partial y}=0, \quad \frac{\partial c}{\partial y}=0
$$

At the out flow boundary side branches $\Gamma_{6}$,

$$
\frac{\partial u}{\partial x}=0, \quad \frac{\partial v}{\partial x}=0, \quad \frac{\partial c}{\partial x}=0
$$

The Local Nusselt number is defined as

$$
N u_{x, t}=\frac{h_{x, t} H}{k}=-\frac{\partial c}{\partial n}
$$

where $\mathrm{k}$ denotes the thermal conductivity of air and $h_{x, t}$ represents the local heat transfer coefficient.

The spatial-averaged Nusselt number on one surface of a heated wall is computed as follows:

$$
N u_{t}=\frac{1}{S} \int_{0}^{s} N u_{x, t} d x
$$

where $\mathrm{S}$ is the length of the heated surface.

To find velocity profile, pressure fields, and temperature profile, the governing equations (1-3) are solved along with the aforementioned boundary conditions (4-9) for fluid flow and heat transfer in a T-channel over a Rotating circular obstacle.

\section{Implementation of the finite element scheme}

The goal is to create a time discretization approach for the equations (1-3). For keeping the essential nonlinearity in the Navier- Stokes equations, we choose an implicit Crank-Nicolson approach as reported in [4]. By re-framing $\mathbf{u}$ to be velocity at current time level and $\mathbf{u}_{\mathbf{1}}$ to be velocity at prior time level using the time-centered Crank-Nicolson technique, we get the following problems:

$$
\begin{gathered}
\nabla \cdot \mathbf{U}=0 \\
\frac{\mathbf{u}-\mathbf{u}_{\mathbf{1}}}{\Delta t}+\mathbf{u}_{\mathbf{1}} \cdot \nabla \mathbf{u}_{\mathbf{1}}=-\nabla p+\frac{1}{R e} \nabla^{2} \mathbf{U} \\
\frac{c-c_{1}}{\Delta t}+\mathbf{U} \cdot \nabla C=\frac{1}{\operatorname{RePr}} \nabla^{2} C
\end{gathered}
$$

with

$$
\mathbf{U}=\frac{1}{2}\left(\mathbf{u}+\mathbf{u}_{1}\right), \quad C=\frac{1}{2}\left(c+c_{1}\right)
$$


The variational formulation that corresponds to the integrals

$$
\begin{gathered}
\int_{\Omega}\left(\left(\frac{\mathbf{u}-\mathbf{u}_{\mathbf{1}}}{\Delta t}\right) v_{u}+\left(\mathbf{u}_{\mathbf{1}} \cdot \nabla \mathbf{u}_{\mathbf{1}}\right) \cdot v_{u}-p \nabla \cdot v_{u}+\frac{1}{R e} \nabla \mathbf{U}: \nabla v_{u}+v_{p} \nabla \cdot \mathbf{U}\right) d x=0 \\
\int_{\Omega}\left(\left(\frac{c-c_{1}}{\Delta t}\right) v_{c}+(\mathbf{U} \cdot \nabla C) \cdot v_{c}+\frac{1}{\operatorname{RePr}}\left(\nabla C \cdot \nabla v_{c}\right)\right) d x=0
\end{gathered}
$$

where $v_{u}, v_{p}$ and $v_{c}$ are test functions for the test spaces of $u, p$, and $c$ respectively.

Mesh creation, assembling the matrix system, and solution of the ensuing systems are the three sub-problems that finite element software has to tackle. Coupled solvers employing MixedFunctionSpace would utilise an entirely different technique to solve for temperature, velocity and pressure at the same time. FEniCS [1] is sophisticated software for finite element modelling with linear algebra solvers like PETSc [16] and Trillinos [15]. The solver is run for a range of time interval $[0,30]$ with time-step $\Delta t=0.001$. A mesh having 57757 vertices and 111935 triangles are shown in figure 2 . To get rid of the larger gradients in the temperature and velocity boundary layer, as well as in the region of the junction, the mesh is more finer near the channel and cylinder walls. The grid sensitivity study of the result obtained with the number of elements and mesh size ensures that an ideal grid distribution is obtained with accurate results and minimal processing time for $\mathrm{Re}=100$ and $\omega=0$ in Table 1 .

\begin{tabular}{|c|c|c|}
\hline $\begin{array}{c}\text { Number of } \\
\text { elements }\end{array}$ & $L_{r} / H$ & $\begin{array}{c}\text { Relative } \\
\text { difference (\%) }\end{array}$ \\
\hline 63559 & 3.0518 & 0.0295 \\
\hline 111935 & 3.0523 & 0.0131 \\
\hline 176443 & 3.0527 & 0.0000 \\
\hline
\end{tabular}

Table 1 Grid sensitivity analysis for $\mathrm{Re}=100$ and $\omega=0$

\subsection{Validation of the findings}

Classical results for the incompressible fluid flow a T-channel reported in the literature $[5,7]$ have been benchmarked with the numerical solution approach utilised here. The length of reattachment has been validated for Newtonian fluids at various Reynolds values is shown in Fig.3. For the results of [5], the least variation for the percentage in error is found to be approximately $0.18 \%$, while the highest deviation is determined to be around $2.37 \%$, whereas the greatest variance percentage, on the other hand, is roughly $1.29 \%$. This verifies the current numerical solution. Overall, there is strong agreement, with slight discrepancies for higher Reynolds numbers in the comparative results. This discrepancy is thought to be related to changes in domain and grid sizes employed by prior studies versus the current one. 


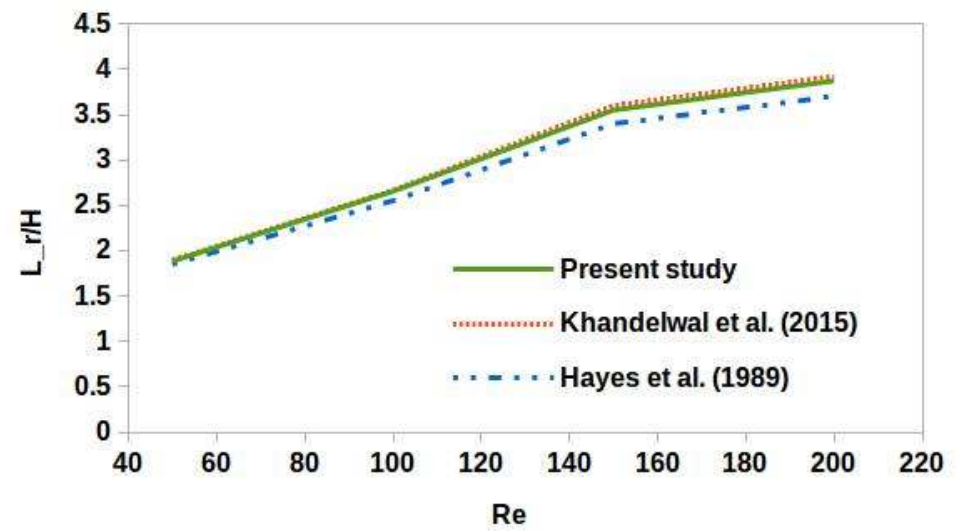

Fig 3: Recirculation length $\left(L_{r} / H\right)$ Vs Reynolds numbers(Re)

\section{$4 \quad$ Results and Discussion}

The key characteristics that determine flow and thermal qualities are the Reynolds number, Prandtl number, cylinder position and diameter, range between the inflow and the junction, cylinder angulation, and expansion ratio. The numerical study for the Reynolds number $(10 \leq R e \leq 200)$ and cylindrical obstacle circumrotation angle $(-5 \leq \omega \leq 5)$ are investigated in the current work.

\subsection{The impact of the cylinder rotation angle}

At a blockage ratio of $20 \%$, the impact of changing the obstacle rotation angle $(\omega)$ on streamlines and isotherms for static $\mathrm{Re}=100$ and the $\mathrm{Pr}=0.71$ is shown in Figs. 4 and 5. The situation $\omega=0$ corresponds to the stationary cylinder depicted in Fig. 4 (b). In the presence of the immobile cylinder, streamlines are somewhat redirected towards the side branch, and many vortices emerge behind the obstruction and near the cylinder close to the side branch's bottom wall. Furthermore, until $\mathrm{Re}=23$, there is no recirculation zoze in the side branch. With a cylinder rotation angle of $\omega$, the flow separation is delayed.

A positive rotation number $(\omega=5)$ implies that the cylinder is rotating counterclockwise Fig.4 (c). Because of the combined impact of contraction area and rotation, more flow is propelled towards the side branch when the cylinder spins in this direction. When compared to a motionless cylinder, the fluid flowing the right side of the obstacle moves more and recirculation zone on the bottom wall of the secondary branch grows. The flow structure around the cylinder's upper and left edges is influenced to some extent. In comparison to the immobile cylinder example, the scale and extent of recirculation zone forming on bottom of the secondary branch risesThe contraction effect accelerates flow in the region between the left wall and the circular cylinder as the cylinder rotates clockwise (negative value of $\omega$ ) Fig. 4(a). Because a portion of the flow is directed to the right of the cylinder, a vortex emerges on the 
upper right of the cylinder, and the immobile cylinder case vanishes. In comparison to the immobile cylinder example, the scale and extent of the recirculation zone forming on the bottom wall of the side branch diminishes. The extent and strength of recirculation region can be regulated using the angle of cylinder rotation, as shown in the figures (Figs.4).

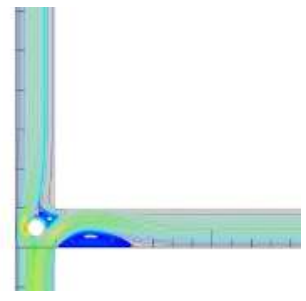

(a) $\omega=-5, t=30$

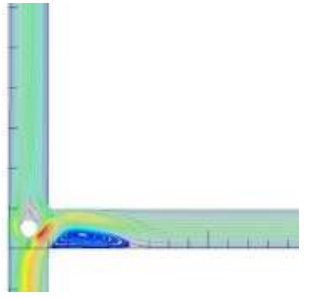

(b) $\omega=0, t=30$

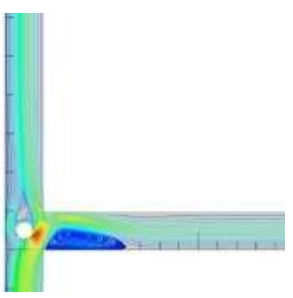

(c) $\omega=5, t=30$

Fig 4: For set value of $\mathrm{Re}=100$, the impact of rotation of the cylinder on streamlines

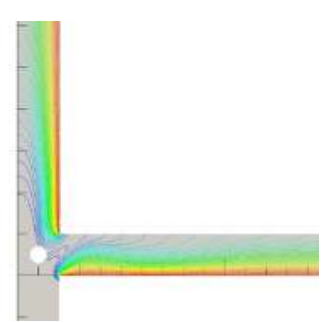

(a) $\omega=-5, t=30$

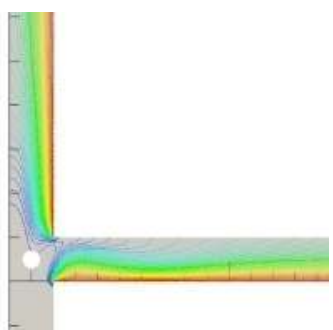

(b) $\omega=0, t=30$

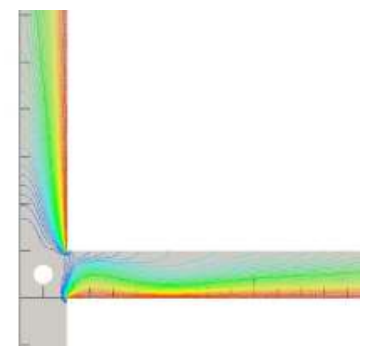

(c) $\omega=5, t=30$

Fig 5: For set value of $\mathrm{Re}=100$, the impact of rotation of the cylinder on isotherms

The contraction impact accelerates flow in the zone between the left side wall and the circular obstacle as the cylinder obstacle rotates clockwise (negative value of $\omega$ ) Fig. 4(a). Because a part of the flow is moves to the right of the obstacle, a vortex emerges on the upper right of the obstacle, and the immobile obstacle case vanishes. In comparison to the immobile cylinder obstacle example, the scale and extent of recirculation zone forming on bottom wall of the secondary branch diminishes. Extent and strength of recirculation region can be regulated using the angle of cylinder rotation, as shown in the figures (Figs.4).

The impact of obstacle rotation on the isotherms for Reynolds number $=100$ is seen in Figs. 5 . Due to the creation of vortices, the isotherms more fluctuate on the secondary branch and bottom regions of obstacle in counter clockwise rotation direction than in the static cylinder example. Because extremely high flow is driven through the space between the primary branch left wall and obstacle as cylinder obstacle rotates clockwise, the isotherms do not cluster as much for the side branch as a result of the recirculation zone occurs, indicating weak heat transfer characteristics for this area. 


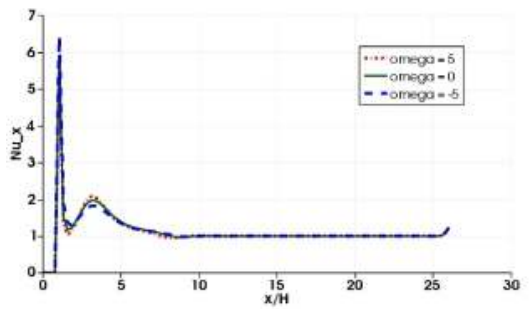

(a) $N u_{x}, \operatorname{Re}=50$

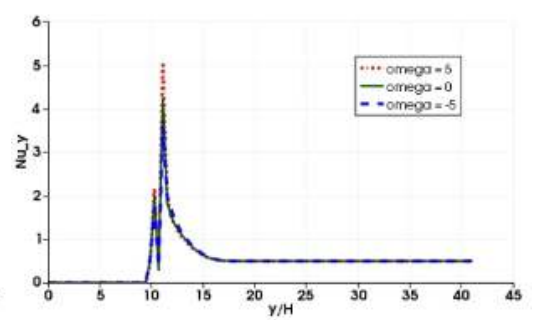

(b) $N u_{y}, \operatorname{Re}=50$

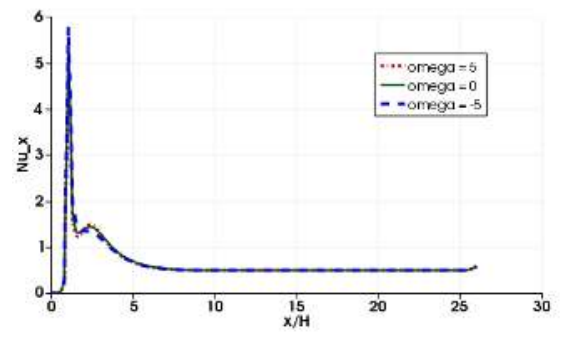

(a) $N u_{x}, \operatorname{Re}=100$

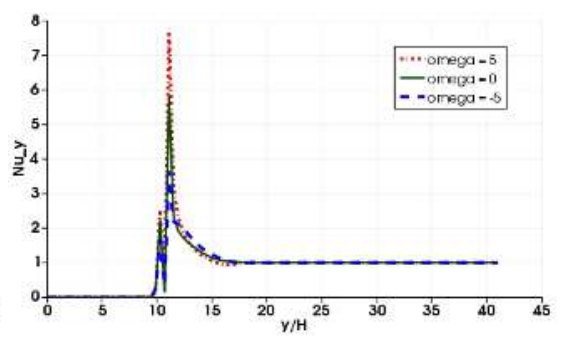

(b) $N u_{y}, \mathrm{Re}=100$

Fig 6: For varied values of $\mathrm{Re}=50,100$, the influence of cylinder angular position on the local Nusselt number distribution

Figures 6 shows local Nusselt number distributions over the surfaces of right side of the primary and the base of the secondary branch. Because of fluid's acceleration toward primary and secondary branches, including a cylinder (motionless or spinning) improves thermal transmission from the right surface of the primary branch and the botom surfaces of the secondary branch. The efficiency of heat transport is lower on the main branch when the cylinder is rotated counterclockwise. Due to the fluid's acceleration toward the primary and secondary branches, poor heat transfer characteristics are observed at the botom surface of the secondary branch during clockwise rotation of obstacle.

\subsection{The impact on Recirculation length}

In Fig.7, the modification of recirculation length $\left(L_{r} / H\right)$ in the secondary branch as a function of Reynolds number and angle of rotation $\omega$ is depicted. With a rise in Reynolds number for a given angular rotation, recirculation zone's distance appears to increase in a non-linear manner. For a given Reynolds number, the recirculation length appears to rise as the angle of rotation decreases. 


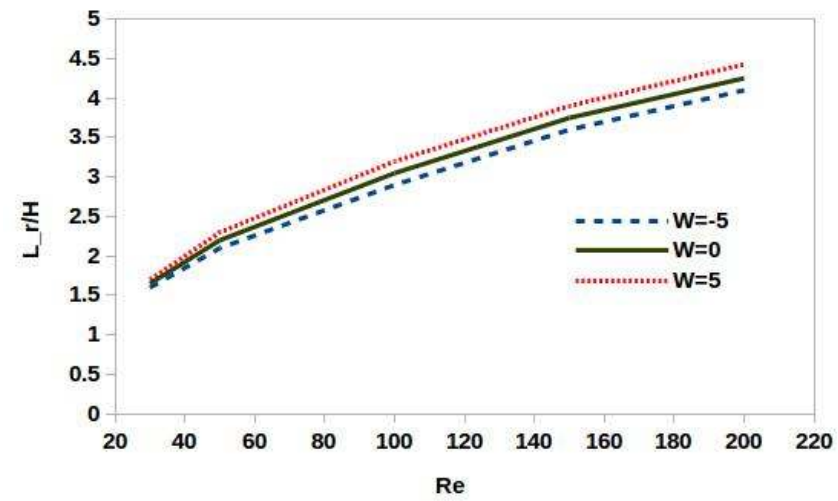

Fig 7: Variation of recirculation zone length $L_{r} / H$ with Re at various level of cylinder rotation angle

\subsection{The influence of Reynolds number}

For particular values of $\omega=-5$ and varied time levels, Figs. 8 and 9 shows variations in Reynolds numbers have an impact on flow and thermal patterns. The fluid maintains touch with the side branch's wall as it travels from the main to side branch.

The fluid separates from bottom wall of the secondary branch beyond a critical point as the Reynolds number increases, and a closed recirculation area develops. The flow resumes its fully developed flow behaviour further downstream of the reattachment locations. The size of recirculation region grows in proportion to the amount of Reynolds number $(\operatorname{Re}>22)$. The flow patterns in a T-channel for Newtonian fluids are found to be quite similar to those observed by Hayes et al. [5] and Neary et al. [11].

The flow separates behind the cylinder as the Reynolds number rises, generating vortex shedding. It's an erratic phenomena in which a recirculation region appears and grows in size and vigour. As seen in Fig 8 (b), some of fluid split from lower wall separates again behind the cylinderical obstruction at a Reynolds number of 100 . When the Reynolds number rises, The flow that has been separated from the lower is induced into the wake of cylinderical obstruction, resulting in the production of vortices behind the cylinderical obstacle Figs.8.

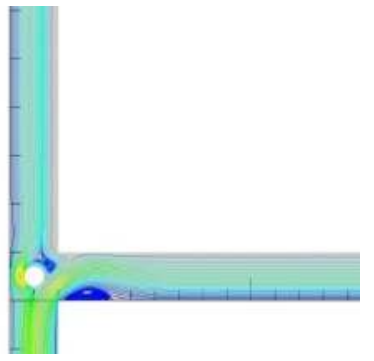

(a) $\mathrm{Re}=50, \mathrm{t}=30$

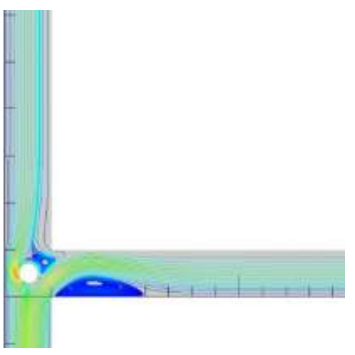

(b) $\mathrm{Re}=100, \mathrm{t}=30$

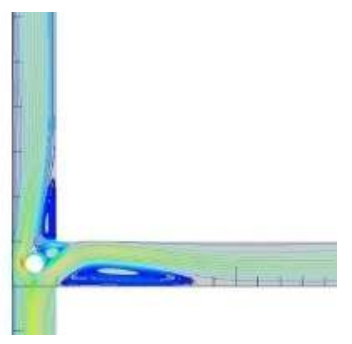

(c) $\mathrm{Re}=200, \mathrm{t}=30$

Fig 8: The impact of Re on the streamlines for $\omega=-5$ 

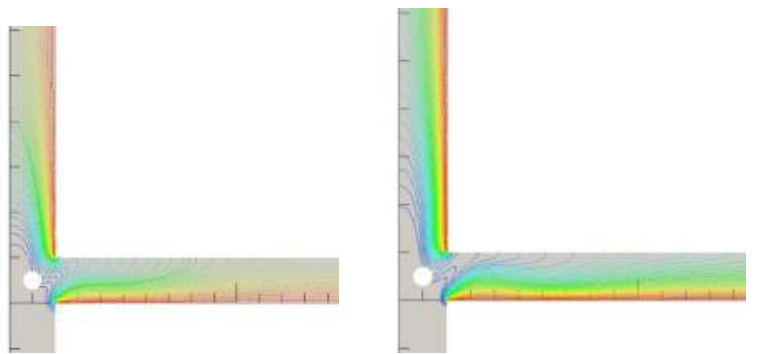

(a)

(b) $\mathrm{Re}=100, \mathrm{t}=30$

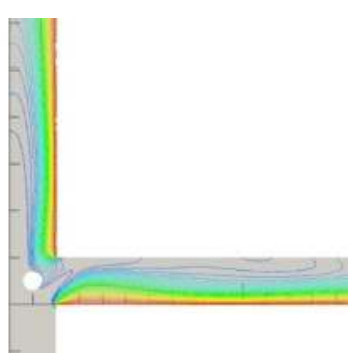

(c) $\mathrm{Re}=200, \mathrm{t}=30$

Fig 9: The impact of $\operatorname{Re}$ on the isotherms for $\omega=-5$

The impact of different Reynolds numbers on isotherms for a set rate of rotation angle $\omega=$ -5 is seen in Figs.9. The isotherms oscillate more on the secondary branch since the obstacle rotates in this direction, and there is fewer clustering of isotherms for the secondary branch because of recirculation zone. Because greater flow moves towards the space between primary left wall and obstacle, it implies that this region has a better heat transfer characteristic

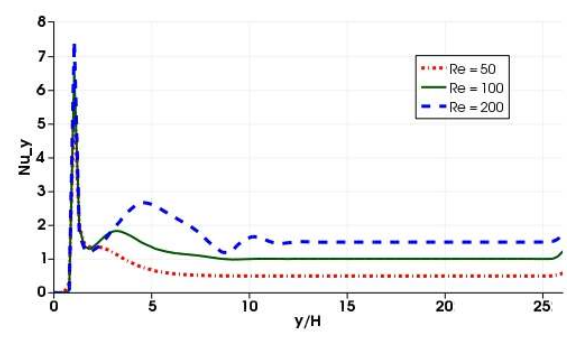

(a) $N u_{x}, \omega=-5$

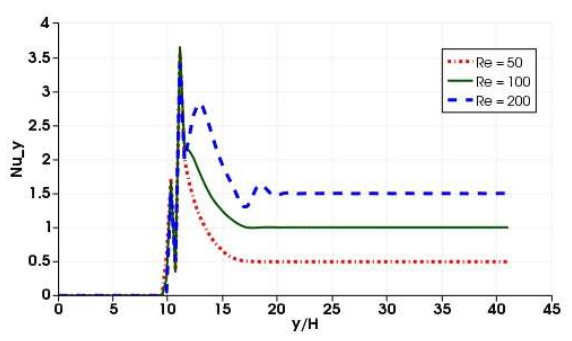

(b) $N u_{y}, \omega=-5$

Fig 10: For a fixed degree of cylinder rotation angle, the impact of $\mathrm{Re}$ on the local $\mathrm{Nu}$ distribution

Figure 10 indicats effect of changing Reynolds number on local Nusselt number for cylinder angular position of $\omega=-5$. It can be deduced from this graph that as the Reynolds number rises, the maximum Nusselt values rise as well. The second highest correlates to reattachment of recirculation zone on the lower boundary of secondary branch at $\mathrm{Re}=100$ and 200. When Reynolds numbers are going down, the impact of cylinder angular position on local Nusselt number dispersion is more evident. With cylinder rotation at $\operatorname{Re}=50$, peak and average heat transmission increase.

\section{Conclusion}

The numerical simulation of a laminar flow field in a T- channel with an adiabatic rotating circular obstacle in the junction is carried. The impact of Reynolds number $(10 \leq R e \leq$ $200)$ and obstacle angular position $(-5 \leq \omega \leq 5)$ on flow and heat transfer properties is explored numerically. 
Previous numerical research have been used to validate the current numerical methodology. After considerable testing with various grid sizes, the grid and computing domain were chosen. The commencement of flow separation along the lower boundary of the side branch and right of the primary branch has been closely examined, including pattern of flow, recirculation zone length, and local Nusselt number.

- The bifurcation creates two recirculation zones, one on the main channel's bottom wall and the other on the upstream wall of the branching channel. The results reveal that as the Reynolds number increases for a given angle of cylinder rotation, the length of the recirculation zone grows.

- A greater amount of flow is entrained into the cylinder's wake as it turns clockwise, and part of it is sent to the right of the cylindrical obstacle. The diameter and length of recirculation bubble that forms on the secondary branch lower wall is modified as a result of these influences. The cylinder rotation angle has a significant impact on the length and severity of recirculation bubble behind the obstruction.

- The isotherm plots are changed when the cylinder obstruction is added. With the installation of the cylinder obstruction, there is a considerable shift in the grouping of isotherm profiles and the location in the flow where this severe temperature gradient emerges. The introduction of a circular cylinder into a T-channel flow has been shown to be successful in changing the velocity profile of the flow if the cylindrical obstacle is positioned correctly.

- On a certain angle of rotation, when Reynolds number rises, local Nusselt number rises as well. Furthermore, it is discovered that when Reynolds number rises, Nusselt number grows monotonically in its maximum and average levels, and that the placement of cylinder obstructions enhances the peak Nusselt values.

\section{Notations}

\begin{tabular}{|c|l|c|l|}
\hline$c$ & temperature, $K$ & $u_{0}$ & inlet fluid velocity, $\mathrm{ms}^{-1}$ \\
\hline$C_{p}$ & specific heat, $J / \mathrm{kgK}$ & $L_{1}$ & main branch upstream length, $m$ \\
\hline$D$ & cylinder diameter, $m$ & $L_{2}$ & downstream length of main branch, $m$ \\
\hline$h$ & coefficient of local heat transfer, $W m^{-2} K^{-1}$ & $L_{3}$ & side branch length, $m$ \\
\hline$H$ & width of the branch, $m$ & $n$ & unit normal vector \\
\hline$k$ & thermal conductivity, $W m^{-1} K^{-1}$ & $N u$ & local Nusselt number, $h H / k$ \\
\hline$R e$ & Reynolds number, $U H / v$ & $\bar{N} u$ & Nusselt number with a spatial-averaged \\
\hline$t$ & time, $s$ & $p$ & pressure, $P a$ \\
\hline$u$ & velocity of the x-component, $m s^{-1}$ & $P r$ & Prandtl number, $\mu C_{p} / k$ \\
\hline$v$ & velocity of the y-component, $\mathrm{ms}^{-1}$ & $\omega$ & cylinder rotation angle. \\
\hline$x, y$ & cartesian coordinates, $m$ & $\rho$ & density, $\mathrm{kgm}^{-3}$ \\
\hline$x_{c}, y_{c}$ & cylinder center location, $m$ & $v$ & kinematic viscosity, $\mathrm{m}^{2} \mathrm{~s}^{-1}$ \\
\hline
\end{tabular}




\section{References}

[1] Logg, K.-A. Mardal and G.N. Wells, Automated Solution of Differential Equations by the Finite Element Method, Springer, 2012.

[2] V. Girault, P.A. Raviart, Finite Element Method for Navier-Stokes Equations: Theory and Algorithms, New York: Springer-Verlag, 1986.

[3] M. F. El-Shaboury, H. M. Soliman, and S. J. Ormiston Laminar forced convection in twodimensional equal-sided and reduced branching ducts, Numer Heat Transfer, Part A, 42, 487-512 (2002).

[4] R. Eymard, T. Gallouet and R. Herbin, Finite Volume Methods, Handbook of Numerical Analysis, Vol.7, 713-1020 (2003). Edited by P.G. Ciarlet and J.L. Lions (North Holland).

[5] R.E. Hayes, K. Nandakumar and H. Nasr-El-Din, Steady laminar flow in a 90 degree planar branch, Comput Fluids 17, 537-553 (1989).

[6] Y. Kawashima, M. Nakagawa and S. Luchi, The Heat Transfer Characteristics of a Twodimensional, Right Angled, T-Shaped Flow Junction, Int. Chem. Eng. vol.23, 510-516 (1983).

[7] V. Khandelwal, A. Dhiman, L. Baranyi, Laminar flow of non-Newtonian shear-thinning fluids in a T-channel, Comput Fluids 108, 79-91 (2015).

[8] J.M. Khodadadi, T.M. Nguyen and N.S. Vlachos, Laminar forced convective heat transfer in a twodimensional $90^{\circ}$ bifurcation, Numer Heat Transfer 9, 677-695 (1986).

[9] Pollard Computer Modeling of Flow in Tee-Junctions, PCH Phys.-Chem. Hydro-dyn. vol.2, 203227 (1981).

[10] N. Moshkin, D. Yambangwi, Steady viscous incompressible flow driven by a pressure difference in a planar T-junction channel, Int J Comput Fluid Dyn 23, 259-270 (2009).

[11] V.S. Neary, F. Sotiropoulos, Numerical investigation of laminar flows through 90-degree diversions of rectangular cross-section, Iowa Institute of Hydraulic Research, The University of Iowa, Iowa City, IA 52242, USA; 1995.

[12] S. Singha, K.P. Sinhamahapatra, Flow past a circular cylinder between parallel walls at low Reynolds numbers, Ocean Eng. 37, 757-769 (2010) .

[13] D. Liepsch and S. Moravec and A.K. Rastogi and N.S. Vlachos, Measurements and calculation of laminar flow in a ninety degree bifurcation, Journal of Biomechanics 15, 473-485 (1982).

[14] R.K. Shah and A.L. London Laminar Flow Forced Convection in Ducts, Academic Press, New York, 1978.

[15] M.A. Heroux and R.A. Bartlett and V.E. Howle and R.J. Hoekstra and J.J. Hu and T.G. Kolda and R.B. Lehoucq and K.R. Long and R.P. Pawlowski and E.T. Phipps and A.G. Salinger and H.K. Thornquist and R.S. Tuminaro and J.M.Willenbring and A. Williams and K.S. Stanley, An overview of the Trilinos project, ACM Transactions Mathematical Software, 31, 397-423 (2005).

[16] S. Balay and J. Brown and K. Buschelman and V.Eijkhout and W.D. Gropp and D. Kaushik and M.G. Knepley and L. Curfman McInnes and B.F. Smith and H. Zhang PETSc users manual, Tech. Report ANL-95/11, Argonne National Laboratory, 2010. 